\title{
Advance ultrasound techniques for the assessment of plaque vulnerability in symptomatic and asymptomatic carotid stenosis: a multimodal ultrasound study
}

\author{
Yi Li ${ }^{1,2}$, Shuai Zheng ${ }^{1,2}$, Jinghan Zhang ${ }^{1,2}$, Fumin Wang ${ }^{1,2}$, Xinyao Liu ${ }^{1,2}$, Wen He ${ }^{1,2}$ \\ ${ }^{1}$ Department of Ultrasound, Capital Medical University, Beijing, China; ${ }^{2}$ Department of Ultrasound, Beijing Tiantan Hospital, Beijing, China \\ Contributions: (I) Conception and design: Y Li, W He; (II) Administrative support: W He; (III) Provision of study materials or patients: Y Li; (IV) \\ Collection and assembly of data: J Zhang, F Wang, X Liu; (V) Data analysis and interpretation: Y Li, S Zheng; (VI) Manuscript writing: All authors; \\ (VII) Final approval of manuscript: All authors. \\ Correspondence to: Wen He, MD. Beijing Tiantan Hospital Affiliated to Capital Medical University, No. 119, South Fourth Ring Road West, Fengtai \\ District, Beijing, China. Email: ttyyus@sina.com.
}

\begin{abstract}
Background: Advanced carotid ultrasound techniques may be useful in characterizing plaque vulnerability, but comprehensive studies are still lacking. The aim of this study was to identify factors associated with vulnerable plaques using advanced ultrasound techniques.
\end{abstract}

Methods: This is a prospective observational study of patients with $>50 \%$ internal carotid stenosis (ICA). All patients underwent conventional ultrasound, superb microvascular imaging (SMI) and shear wave elastography (SWE) examinations. Plaque size, echogenicity, stiffness and intraplaque neovascularization (IPN) were assessed and compared between symptomatic and asymptomatic groups. Receiver operating characteristic (ROC) curves were used to evaluate the diagnostic performance of SWE and SMI of the vulnerable plaques.

Results: The final analysis included 123 patients (78.9\% male; mean age, $66 \pm 8$ years), 65 were enrolled in the symptomatic group, and 58 were enrolled in the asymptomatic group. The mean elasticity was $78.1 \pm 25.4 \mathrm{kPa}$ for asymptomatic and $51.5 \pm 18.3 \mathrm{kPa}$ for symptomatic plaques. Symptomatic plaques showed higher visual IPN grades on SMI than asymptomatic plaques $(\mathrm{P}<0.001)$. Multivariate regression analysis showed that plaque stiffness (PS) (OR 0.95, 95\% CI, 0.919-0.974) and IPN level (OR 4.17, 95\% CI, 2.008-8.664) were independently associated with symptomatic plaques. The combination of the two factors had a preferable accuracy to discriminate symptomatic plaques (AUC 0.89, 95\% CI, 0.827-0.944).

Conclusions: Advanced carotid ultrasound techniques can identify plaque characteristics that are associated with ischemic events and may be potentially indicative of plaque vulnerability. These factors may ultimately be used in the clinical management of carotid stenosis.

Keywords: Carotid plaque; vulnerability; shear wave elastography (SWE); superb microvascular imaging (SMI); stroke

Submitted Oct 24, 2020. Accepted for publication Dec 14, 2020.

doi: $10.21037 / \mathrm{cdt}-20-876$

View this article at: http://dx.doi.org/10.21037/cdt-20-876

\section{Introduction}

Stroke is a public health problem and is the leading cause of death and disability worldwide (1). Current guidelines for stroke prevention in patients with carotid plaques are based on the severity of lumen narrowing, and this factor is important for decision making to prevent stroke occurrence $(2,3)$. Mounting evidence shows that vulnerable plaques are more likely to lead to ischemic events, independent of the severity of carotid stenosis $(4,5)$. Therefore, risk stratification of patients with asymptomatic carotid 
stenosis is necessary to improve target patient selection for interventional therapies, and carotid plaque vulnerability assessment may help fulfill this goal.

Atherosclerotic plaques associated with a high likelihood of neurological event occurrence and those that progress rapidly should be considered vulnerable plaques (6). Based on histopathological findings, several key plaque composition characteristics such as a thin fibrous cap, a large lipid rich necrotic core, intraplaque hemorrhage (IPH), inflammatory and intraplaque neovascularization (IPN), are associated with vulnerable plaques. New imaging techniques that can identify vulnerable carotid plaques in vivo are therefore needed for more accurate targeting of prophylactic therapy and preventing the occurrence of stroke.

Carotid ultrasound is a noninvasive and widely used imaging approach for carotid plaque (atherosclerosis) screening. Conventional ultrasound techniques have enabled routine characterization of carotid plaque features (e.g., in plaque area, echogenicity and surface morphology) (7). Shear wave elastography (SWE) is an emerging ultrasoundbased technique that enables quantitative and noninvasive assessment of tissue stiffness (8). Elastography ultrasound has become increasingly important for differentiation of the characteristics of solid tumors, especially in the breast, liver, and thyroid (9). SWE provides additional information related to plaque characteristics such as the presence of a large lipid core and IPH, which may indicate plaque vulnerability $(10,11)$. However, currently, most of the studies on the assessment of carotid plaque vulnerability by elastography have been performed on small samples of patients. IPN, a well-known marker in plaque destabilization, can be assessed by contrast-enhanced ultrasound (CEUS) as contrastagent enhancement in the plaque (12). However, CEUS is a minimally invasive examination that requires an intravenous injection of a contrast-agent with a related risk that limits its use in clinical practice. Superb microvascular imaging (SMI) is a novel ultrasound technique devised for overcoming the limitations of conventional Doppler ultrasound, which can successfully visualize the microvascular blood flow signals without the use of contrast agents (13).

Our postulate is that plaque composition characteristics, such as IPN and plaque stiffness (PS), will be significantly difference in plaques that cause ipsilateral neurological symptoms compared to asymptomatic plaques. Accordingly, we hypothesize that IPN detected by SMI without using contrast agent is a surrogate mark of plaque vulnerability and that SWE provides more additional information about plaque vulnerability, and stroke risk.

We present the study in accordance with the STARD reporting checklist (available at http://dx.doi.org/10.21037/ cdt-20-876).

\section{Methods}

Our present study was approved by the Institutional Review Board of Beijing Tiantan Hospital (IRB No. KY2019-113-01). All participants gave written informed consent before SWE and SMI examinations. The study was conducted in accordance with the Declaration of Helsinki (as revised in 2013).

\section{Study population}

In this prospective observational study, patients with $>50 \%$ internal carotid artery stenosis, as determined by carotid duplex ultrasound, were consecutively included at Beijing Tiantan Hospital, from December 2018 to February 2020. This population was separated into two groups: symptomatic with an ipsilateral carotid-related ischemic event (amaurosis fugax, transient ischemic attack, or stroke) within the past 30 days, and asymptomatic without any ischemic events in the corresponding carotid territory within the past 6 months. This classification based on ischemic events was used as a surrogate marker of plaque vulnerability. The exclusion criteria were patients who received previous radiation therapy in the neck, patients diagnosed with nonatherosclerotic carotid artery stenosis, patients with $>70 \%$ ipsilateral intracranial artery stenosis confirmed by CTA or MRA, patients with severe disturbance of consciousness, and those in whom plaque visualization was difficult because of an acoustic shadow due to severe calcification. A neurologist who was blinded to the ultrasound findings recorded clinical data from all participants, including age, sex, body mass index (BMI), risk factors for atherosclerosis, history of cardiovascular disease, use of medications, and ischemic events. All participants underwent conventional ultrasound, SMI, and SWE of the carotid arteries before CEA during hospitalization or at a routine outpatient follow-up visit for asymptomatic carotid stenosis.

\section{Conventional ultrasound protocol}

All imaging was performed using the same ultrasound machine (Aplio 900, Canon Medical Systems Corporation, Japan), and a 14L5 (frequency range, 5-14 MHz) linear 


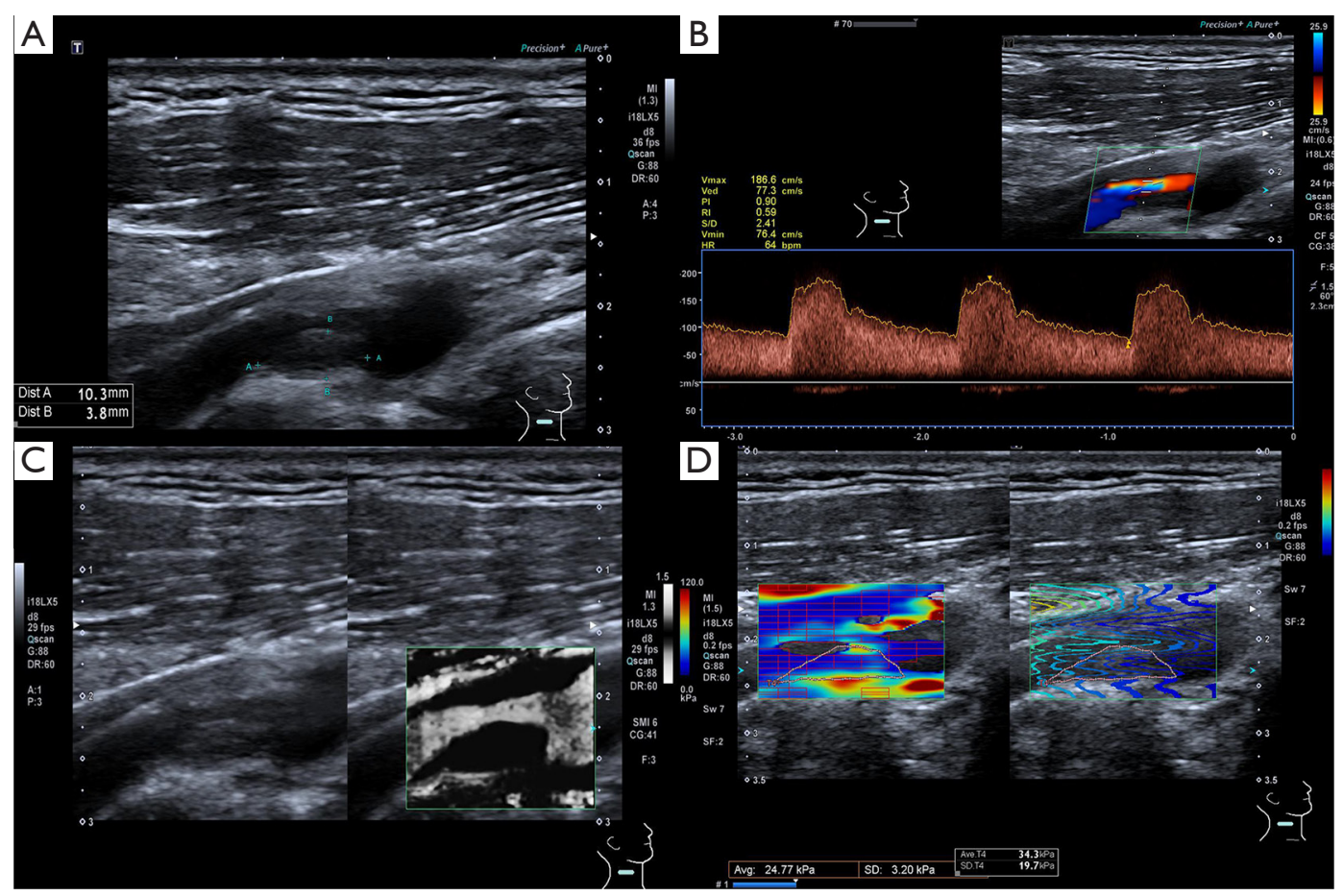

Figure 1 Example of a multimodal ultrasound study. (A) Grayscale ultrasound showed a predominantly hypoechoic plaque located at posterior wall of right proximal internal carotid artery. (B) Pulsed wave Doppler ultrasound showed a strong increase of peak systolic velocity caused by a hemodynamically significant stenosis. (C) SMI in longitudinal view confirmed the high-grade stenosis; no ulcerations were seen along the border of the plaque; no IMVF is visible within the plaque. (D) SWE showed the Young's modulus of the plaque was $34.3 \mathrm{kPa}$, quality control map confirmed that the sampling was done correctly. IMVF, intraplaque microvascular flow; SMI, superb microvascular imaging; SWE, shear wave elastography.

array probe was used for the conventional ultrasound, SMI and SWE examinations (Figure 1). With the patient in the supine position, the bilateral carotid arteries (including the common carotid artery, carotid bifurcation, and internal carotid artery) were scanned in longitudinal and transverse sections in standard carotid ultrasound. All images were independently assessed by two vascular sonographers with five years' experience who were blinded to clinical outcomes.

The degree of internal carotid artery stenosis was graded as follows: $50-69 \%$ and $70-99 \%$ determined based on peak-systolic and end-diastolic velocities according to the ultrasound consensus criteria (14). A carotid plaque was defined as an intima-media thickness greater than $1.5 \mathrm{~mm}$ or a focal wall thickening of at least $50 \%$ greater than the surrounding vessel wall (15). The maximal internal carotid plaque thickness (MICPT), plaque size (area), presence of calcification (yes or no) and plaque echogenicity were collected. Plaque features were assessed in longitudinal sections, and on an image showing the thickest plaque, the intimal-medial wall thickness was measured as the MICPT. In the same image, a plaque was manually delineated and recorded as the plaque size. Plaque echogenicity was classified visually into four groups as follows (16): type 1, uniformly hypoechoic; type 2, predominantly hypoechoic; type 3 , predominantly hyperechoic; and type 4 , uniformly hyperechoic.

\section{SMI protocol}

After the conventional ultrasound examination, the setting of the ultrasound scanner was switched to monochrome SMI mode to show a double display of the plaque of interest in grayscale mode and monochrome SMI mode side-byside. After starting the proprietary monochrome SMI mode, the SMI specific region of interest box was placed around the whole plaque/stenosis. Other SMI technical parameters were modified as follows: mechanical index of 1.5 , frame 
rate of 50-60 fps, dynamic range of 55-60 dB, and SMI velocity range of $1.0-2.0 \mathrm{~cm} / \mathrm{s}$. Plaques were first observed in the transverse section and then in the longitudinal section for 2 minutes, and the dynamic video images were stored on the device hard disk. Moving enhancements were defined as intraplaque microvascular flow (IMVF), and static enhancements (considered to be tissue acoustic reflector) were excluded. IMVF levels were classified on a visual scale according to the previous study proposed by Mahtab Zamani as follows: grade 0, no IMVF or IMVF confined to the adjacent adventitia; grade 1, moving IMVF confined to the adventitial side; grade 2, moving IMVF at the plaque shoulder; grade 3, IMVF moving to the plaque core; and grade 4, extensive IMVF (17). SMI assessments were carried out in forty plaques from grade 0 to grade 4 IMVF on 2 occasions, which were $>2$ weeks apart to obtain the intra- and interobserver variability using $\kappa$ statistics.

\section{SWE protocol}

SWE was performed after the SMI examination with the same ultrasound unit; to obtain a twin-view display of the plaque of interest on the elastographic map and quality control map, inbuilt elasticity software was used. The ultrasound scanner both generated and estimated the propagation speed of the resulting shear waves in order to calculate the Young's modulus ( $\mathrm{YM}, \mathrm{kPa}$ ), based on the formula $\mathrm{YM}=\rho \mathrm{c} 2$, where $\mathrm{YM}$ is tissue elasticity, $\rho$ is tissue density and $c$ is shear wave velocity. The scanner SWE settings were optimized based on initial findings as follows: resolution $=3$; smoothing $=3$; FR control $=3$; and focus $=75 \%$. The shear wave scale was set at a range from $0 \mathrm{kPa}$ to $120 \mathrm{kPa}$. The $\mathrm{SWE}$ specific region of interest box was adjusted to include the entire carotid plaque (typically $1 \mathrm{~cm}$ wide $\times 2 \mathrm{~cm}$ long) (18). With the carotid plaque in a maximal longitudinal view, the SWE procedure was started. The operator placed the probe on the skin without pressure in order to minimize pressure artifact and kept the imaging stable for approximately $3 \mathrm{~s}$ to allow elasticity measurements. This imaging system displays the elasticity in real time by means of a colorimetric map within the elastographic rectangular box. The measurement was manually delineated around the entire plaque. Then, the quantitative elasticity of the plaque was available. The imaging system also generates a quality control map that represents the propagation of the shear wave as wavefront lines. When the propagation lines are parallel to each other, the measurement may be more reliable and accurate; otherwise, if the propagation lines are twisted or missing, repeated measurements may be needed. SWE assessments were carried out in forty plaques to obtain the interobserver variability using the intraclass correlation coefficient (ICC).

Conventional ultrasound images, SMI and SWE frames and cine loops were stored in the local picture archive and communication system (PACS).

\section{Statistical analysis}

All statistical analyses were performed using SPSS 22.0 software (IBM Corporation, New York, USA). Continuous variables are expressed as the means and standard deviations, while categorical variables are expressed as frequencies and percentages. Univariate analysis was performed using a $t$-test for continuous variables and a $\chi^{2}$ test for categorical variables. Multivariate analysis was performed using binary logistic regression models with plaques associated with ischemic symptoms in patients as the dependent variable and plaque characteristics (area, echogenicity, IPN grades, and PS) as independent variables. Variables with $\mathrm{P}<0.10$ in the univariate analysis were included in the models. Receiver operating characteristic (ROC) curves and the area under the curve (AUC) were used to evaluate the clinical discrimination potential. Delong test was used to compare different AUC. A P value $<0.05$ was considered statistically significant.

\section{Results}

\section{Patient characteristics}

We included 169 patients with $>50 \%$ internal carotid artery stenosis during the study period: 9 patients with severe stroke, 4 patients who received neck radiotherapy, 20 patients without MRA or CTA above the aortic arch, 4 patients diagnosed with carotid artery dissection, 4 patients diagnosed with Takayasu's arteritis, and 5 patients with poor ultrasound imaging quality were excluded. Finally, 123 patients, including 97 men $(65 \pm 8$ years $)$ and 26 women $(67 \pm 8$ years $)$ who had complete conventional ultrasound, SMI and SWE data were eligible; 65 of these participants had symptomatic plaques, whereas the rest had asymptomatic plaques.

The baseline characteristics of the included patients are listed in Table 1. Symptomatic and asymptomatic groups did not significantly differ with respect to demographics or medications used. Hypertension was more frequent (but 
not significantly) in asymptomatic group, and dyslipidemia was more prevalent in symptomatic group $(\mathrm{P}=0.026)$. Fiftysix $(45.5 \%)$ patients had moderate stenosis, and sixtyseven patients had severe stenosis. Symptomatic plaques more commonly had severe carotid stenosis, whereas asymptomatic plaques more commonly had moderate carotid stenosis $(\mathrm{P}<0.001)$.

\section{Plaque features}

The mean plaque thickness and mean plaque area were $0.38 \pm 0.08 \mathrm{~cm}(0.23-0.58 \mathrm{~cm})$ and $0.61 \pm 0.21 \mathrm{~cm}^{2}(0.21-$

Table 1 Baseline characteristics of the included patients

\begin{tabular}{lccc}
\hline Characteristics & $\begin{array}{c}\text { Symptomatic } \\
(\mathrm{n}=65)\end{array}$ & $\begin{array}{c}\text { Asymptomatic } \\
(\mathrm{n}=58)\end{array}$ & $\mathrm{P}$ \\
\hline Age, (mean $\pm \mathrm{SD}$ ), year & $66 \pm 8$ & $65 \pm 7$ & 0.388 \\
Man sex & $73.8 \%$ & $70.7 \%$ & 0.695 \\
Body mass index, & $24.3 \pm 2.1$ & $25.1 \pm 3.7$ & 0.113 \\
(mean \pm SD) & & & \\
Previous or current smoker & $73.8 \%$ & $72.4 \%$ & 0.858 \\
Previous or current drinker & $75.4 \%$ & $72.4 \%$ & 0.708 \\
Hypertension & $70.8 \%$ & $77.6 \%$ & 0.391 \\
Diabetes mellitus & $26.2 \%$ & $25.9 \%$ & 0.971 \\
Dyslipidemia & $49.2 \%$ & $29.3 \%$ & 0.026 \\
Coronary artery disease & $32.3 \%$ & $25.9 \%$ & 0.434 \\
Antiplatelet & $78.5 \%$ & $69.0 \%$ & 0.233 \\
Statin & $86.2 \%$ & $77.6 \%$ & 0.220 \\
\hline
\end{tabular}

$1.11 \mathrm{~cm}^{2}$ ), respectively, with no significant differences between symptomatic and asymptomatic groups. The proportions of different plaque echogenicities including uniformly hypoechoic plaques, predominantly hypoechoic plaques, predominantly hyperechoic plaques, and uniformly hyperechoic plaques were $8.9 \%$ (11/123), 41.5\% (51/123), $30.1 \%(37 / 123)$, and $19.5 \%$ (24/123), respectively. The percentage of calcified plaques was $55.3 \%$ (68/123), and that of noncalcified plaque was $44.7 \%$ (55/123). IMVF signals on SMI were found in 104 (84.6\%) of the 123 plaques. On SMI imaging, 19 (15.4\%) plaques had no IMVF signals, $38(30.9 \%)$ plaques had IMVF signals confined to the adventitial side, 42 (34.1\%) plaques had IMVF signals at the plaque shoulder, 22 (17.9\%) plaques had IMVF signals moving to the plaque core, and $2(1.6 \%)$ plaques were found to have extensive IMVF signals. Significant differences were observed between the symptomatic and asymptomatic groups with respect to the different grades of IMVF $(\mathrm{P}<0.001)$ (Figure $2 A)$. The mean PS was $64.2 \pm 25.7 \mathrm{kPa}$, ranging from 23.2 to $126.4 \mathrm{kPa}$. Symptomatic plaques $(51.5 \pm 18.3 \mathrm{kPa})$ were softer than asymptomatic plaques $(78.5 \pm 25.4 \mathrm{kPa})(\mathrm{P}<0.001)$ (Figure $2 B)$. The mean $\mathrm{YM}$ of plaques in the moderate stenosis (50-69\%) group was $72.3 \pm 25.5 \mathrm{kPa}$, and that in the severe stenosis $(70-99 \%)$ group was $57.5 \pm 23.8 \mathrm{kPa}(\mathrm{P}<0.001)$ (Figure $2 C)$. Carotid plaque features and comparisons between symptomatic and asymptomatic groups are summarized in Table 2.

The intra- and interobserver variability for the assessment of IPN using the 5-level category with SMI was favorable, with kappa coefficients of 0.778 and 0.705 , respectively, between the 2 independent sonographers. The interrater variability for the assessment of PS using SWE
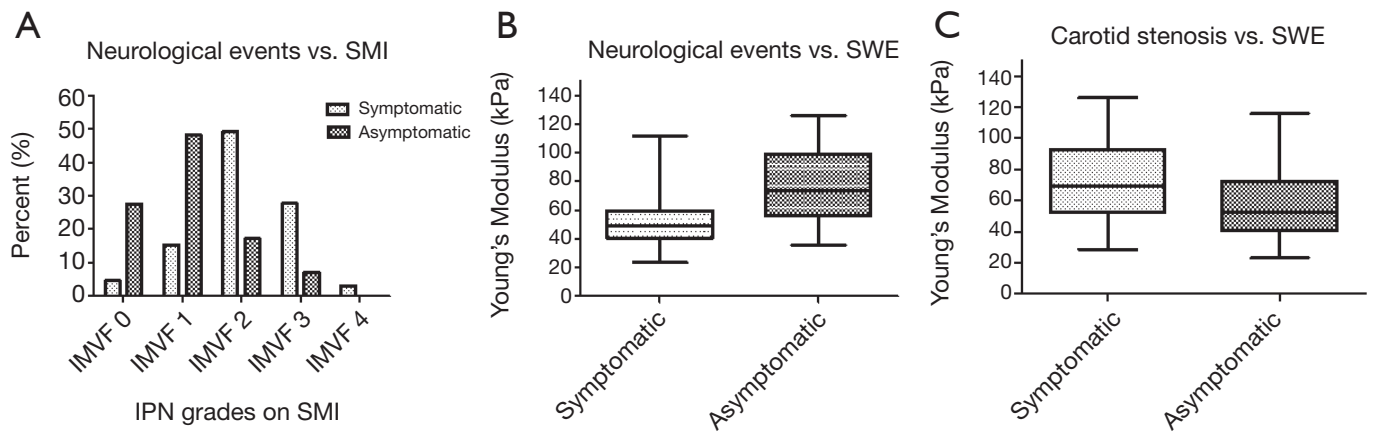

Figure 2 Graphs illustrating key findings of our study. (A) Distribution of visualization IPN grades between symptomatic and asymptomatic patients. (B) The relationship between plaque YM and neurological events, grouped into symptomatic and asymptomatic. (C) Plaque YM against the degree stenosis, grouped into either moderate (50-70\%) or severe (70-99\%). IPN, intraplaque neovascularization; IMVF, intraplaque microvascular flow; YM, Young's modulus; SMI, superb microvascular imaging; SWE, shear wave elastography. 
Table 2 Carotid plaque features and their comparison between symptomatic and asymptomatic patients

\begin{tabular}{|c|c|c|c|c|c|c|}
\hline Characteristics & $\begin{array}{l}\text { Symptomatic } \\
\qquad(n=65)\end{array}$ & $\begin{array}{l}\text { Asymptomatic } \\
\qquad(n=58)\end{array}$ & Univariate analysis $\mathrm{P}$ & \multicolumn{3}{|c|}{ Multivariate analysis } \\
\hline MICPT (cm) & $0.38 \pm 0.07$ & $0.39 \pm 0.09$ & 0.344 & & & \\
\hline Plaque area $\left(\mathrm{cm}^{2}\right)$ & $0.65 \pm 0.19$ & $0.58 \pm 0.22$ & 0.067 & 3.05 & $0.25-37.46$ & 0.384 \\
\hline Calcified plaque & $53.8 \%(n=35)$ & $56.9 \%(n=33)$ & 0.734 & & & \\
\hline Uniformly hypoechoic & $9.2 \%(n=6)$ & $8.6 \%(n=5)$ & & & & \\
\hline Predominantly hypoechoic & $49.2 \%(n=32)$ & $32.8 \%(n=19)$ & & & & \\
\hline Predominantly hyperechoic & $24.6 \%(n=16)$ & $36.2 \%(n=21)$ & & & & \\
\hline Uniformly hyperechoic & $16.9 \%(n=11)$ & $22.4 \%(n=13)$ & & & & \\
\hline Grade 1 & $15.4 \%(n=10)$ & $48.3 \%(n=28)$ & & & & \\
\hline Grade 2 & $49.2 \%(n=32)$ & $17.2 \%(n=10)$ & & & & \\
\hline Grade 3 & $27.7 \%(n=18)$ & $6.9 \%(n=4)$ & & & & \\
\hline Grade 4 & $3.1 \%(n=2)$ & 0 & & & & \\
\hline $\mathrm{PS}, \mathrm{kPa}$ & $51.5 \pm 18.3$ & $78.5 \pm 25.4$ & $<0.001$ & 0.95 & $0.93-0.97$ & $<0.001$ \\
\hline Degree of stenosis & & & $<0.001$ & 5.27 & $1.79-15.55$ & 0.003 \\
\hline $50-69 \%$ & $26.2 \%$ & $67.2 \%$ & & & & \\
\hline $70-99 \%$ & $73.8 \%$ & $32.8 \%$ & & & & \\
\hline
\end{tabular}

OR, odds ratio; $\mathrm{Cl}$, confidence interval; MICPT, maximal internal carotid plaque thickness; IPN, intraplaque neovascularization; PS, plaque stiffness.

was found to have an ICC $=0.911$ (95\% CI, 0.848-0.948) between the 2 independent sonographers.

\section{Factors independently associated with symptomatic plaques}

Among variables with a univariate $\mathrm{P}<0.1$, dyslipidemia, the degree of stenosis, plaque area, IPN level, and PS were included in the multivariate model. The degree of stenosis (OR 5.27, 95\% CI, 1.79-15.55, P=0.003), IPN level (OR 4.19, 95\% CI, 2.09-8.40, $\mathrm{P}<0.001$ ) and PS (OR 0.95, 95\% CI, 0.93-0.97, $\mathrm{P}<0.001$ ) were independently associated with symptomatic plaques (Table 2).

\section{ROC curve analysis}

The accuracy of differentiating symptomatic plaques from asymptomatic plaques were assessed using carotid stenosis degree, IPN level, PS and the combination of IPN level and PS (Figure 3). The combination of the IPN level and PS yielded the best agreement to classify symptomatic plaques (AUC $=0.89,95 \%$ CI, 0.827-0.944). Delong test showed that the combination of IPN level and PS model was significantly superior to that of the other three factors alone (all $\mathrm{P}<0.05$ ). For SWE, SMI, and their combination, the sensitivity was $84.5 \%, 80 \%$ and $76.9 \%$, respectively, and the specificity was $70.8 \%, 75.9 \%$ and $87.9 \%$, respectively, for the identification of symptomatic plaques.

\section{SMI and SWE in moderate carotid stenosis}

To explore the relationship of the SMI and SWE with symptomatic plaques in patients where additional information to carotid stenosis severity might be most useful for clinical management relating to carotid intervention, we elaborated our analyses after exclusion of patients with high-grade carotid stenosis. The subgroup with moderate 


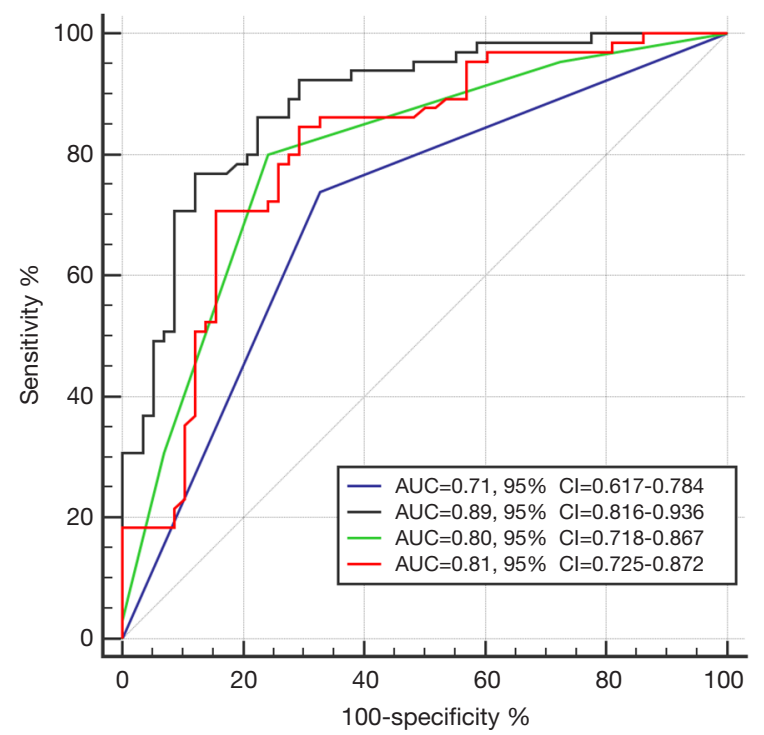

Figure 3 Receiver operating characteristic (ROC) curves. The agreement of differentiating symptomatic plaque from asymptomatic plaque was measured using carotid stenosis degree, IPN level, PS and the combination of IPN level and PS: ROC curve of carotid stenosis degree (blue line), IPN level (green line), PS (red line), and IPN level plus PS (black line). The combination of IPN level and PS (black line) yielded the best accuracy. IPN, intraplaque neovascularization; PS, plaque stiffness; AUC, area under the ROC curve; CI, confidence interval.

carotid stenosis included 56 patients, with 17 symptomatic plaques.

On univariate logistic regression analysis, hypertension, IPN level, and PS were included in the multivariate model. The IPN level (OR 3.4, 95\% CI, 1.36-8.45, P=0.009) and PS (OR 0.95, 95\% CI, 0.91-0.99, P=0.019) were independently associated with symptomatic plaques. On ROC analysis, the AUC of IPN level, PS, and their combination were 0.79 (95\% CI, 0.66-0.92), 0.82 (95\% CI, $0.71-0.95$ ), and 0.87 (95\% CI, 0.78-0.96), respectively.

\section{Discussion}

In clinical practice, the most widely accepted predictor of stroke is the degree of lumen narrowing in carotid atherosclerosis. However, among asymptomatic patients, carotid stenosis degree cannot provide sufficient evidence for identification the risk of stroke, so additional imaging characteristics for assessing plaque composition are becoming significant factors (19). Therefore, only a comprehensive assessment of the plaque features can more precisely stratify patients of stroke risk and guide target treatment. We summarize findings of the existing studies in Table 3. No study has, on the other hand, systematically investigated the association of IPN level or PS with symptomatic plaques in such large sample size, however.

Our study confirms the clinical potential of advanced ultrasound techniques including SWE and SMI to identify high-risk carotid plaques and to differentiate symptomatic from asymptomatic plaques. Plaques with higher SMI grades were more prevalent among symptomatic plaques than among asymptomatic plaques. On SWE imaging, symptomatic plaques were significantly softer than asymptomatic plaques. Multivariate analysis revealed that IPN grades and PS were independently associated with symptomatic plaques. The combination of these two factors differentiated symptomatic plaques from asymptomatic plaques better than each factor alone. These findings suggested that the addition of advanced ultrasound techniques with conventional ultrasound improved diagnostic performance for the detection of vulnerable carotid plaques in patients with asymptomatic carotid stenosis.

The presence of newly generated small blood vessels arising from the adventitia within atherosclerotic lesions leading to IPH plays a key role in plaque destabilization and is therefore associated with neurological symptoms (24). Recently, visualization of the adventitial vasa vasorum and IPN has emerged as a new potential surrogate marker for vulnerable plaques (25). Conventional Doppler techniques filter out low-velocity signals, preventing the visualization of neovascularization. CEUS has shown preferable ability in the visualization of microvessels; however, it is a minimally invasive examination and requires intravenous injection of ultrasound contrast agents $(12,26)$.

SMI is a novel noninvasive technique that can successfully detect microvascular blood flow signals without using ultrasound contrast agents (20). Previously published study showed good agreement between CEUS and SMI in the assessment of IPN (21). Zamani et al. (17) reported that plaques with higher IPN grades had higher numbers of neovessels quantified at histology $(\mathrm{P}=0.041, \mathrm{r}=0.460)$. In their research, however, there was no statistically significant difference between symptomatic and asymptomatic patients with regard to the different levels of IMVF on SMI, which may be because of the relatively small sample size. In our present large-sample study, significant differences were observed between the symptomatic and asymptomatic 
Table 3 Summary of previous studies on SMI and SWE of patients with carotid atherosclerosis

\begin{tabular}{|c|c|c|c|c|}
\hline Article & Setting & Technique & Sample size, $n$ & Main findings \\
\hline (11) & $\begin{array}{l}\text { Patients with ischemic events } \\
\text { who underwent carotid } \\
\text { endarterectomy }\end{array}$ & SWE & 25 & $\begin{array}{l}\text { The mean YM of vulnerable plaques was significantly lower } \\
\text { than that of stable plaques ( } 50.0 \text { vs. } 79.1 \mathrm{kPa} ; \mathrm{P}=0.027) \text {. The } \\
\text { presence of plaque hemorrhage, thrombus and increasing } \\
\text { numbers of foam cells was also associated with a significantly } \\
\text { lower YM }\end{array}$ \\
\hline (13) & $\begin{array}{l}\text { Patients with a carotid } \\
\text { plaque (intima-media } \\
\text { thickness } \geq 2 \mathrm{~mm} \text { ) }\end{array}$ & SMI and CEUS & 27 & $\begin{array}{l}\text { Intraplaque enhancement was observed in } 19 \text { patients using } \\
\text { CEUS, while intraplaque microvascular flow level signals were } \\
\text { observed in } 12 \text { patients using SMI. A } 100 \% \text { specificity was } \\
\text { recorded for SMI, while its sensitivity was } 63 \%\end{array}$ \\
\hline (20) & $\begin{array}{l}\text { Patients with }>50 \% \text { carotid } \\
\text { stenosis who underwent } \\
\text { carotid endarterectomy }\end{array}$ & SMI & 28 & $\begin{array}{l}\text { There was a significant correlation between the density of } \\
\text { neovascularization in histopathologic plaques and the } \\
\text { intraplaque microvascular flow level found by SMI. A significant } \\
\text { difference was observed in SMI intraplaque microvascular flow } \\
\text { level between the symptomatic and asymptomatic groups }\end{array}$ \\
\hline (21) & $\begin{array}{l}\text { Patients with ischemic events } \\
\text { who had a carotid plaque }\end{array}$ & SMI and CEUS & 82 & $\begin{array}{l}\text { Patients after } 6 \text { months atorvastatin treatment, SMI-detected } \\
\text { intraplaque neovascularization reduced from } 69.23 \% \text { to } \\
48.72 \% \text {, while CEUS-detected ones reduced from } 76.92 \% \text { to } \\
69.23 \% \text {. The consistency between CEUS and SMI was above } \\
0.75 \text { at all assessments }\end{array}$ \\
\hline (23) & $\begin{array}{l}\text { Patients with carotid stenosis } \\
>30 \%\end{array}$ & SWE & 54 & $\begin{array}{l}\text { Plaques associated with ischemic symptoms had significantly } \\
\text { lower mean YM than plaques in asymptomatic patients ( } 62 \text { vs. } \\
88 \mathrm{kPa} \text { ). ROC analysis demonstrated improvements in } \\
\text { sensitivity and specificity when percentage stenosis was } \\
\text { combined with the YM (AUC }=0.78 \text { ) }\end{array}$ \\
\hline
\end{tabular}

NIVE, non-invasive vascular elastography; SWE, shear wave elastography; YM, Young's modulus; SMI, superb microvascular imaging; CEUS, contrast-enhanced ultrasound; IPN, intraplaque neovascularization; AUC, area under curve; ROC, receiver operating characteristic.

patients with respect to the different grades of IMVF using SMI $(\mathrm{P}<0.001)$. Only three $(15.8 \%)$ among 19 patients with non-neovascularized plaques had neurological symptoms; however, 62 (59.6\%) of 104 patients with neovascularized plaques had neurological symptoms $(\mathrm{P}<0.001)$. In a prospective study, the authors reported that SMI level was also a preferable marker in predicting the occurrence of future stroke with an AUC of 0.878 (22). These findings demonstrated that IPN detected by SMI plays a key role in plaque vulnerability and the occurrence of stroke, and 
plaque neovascularization may be a potential intervention target for stroke prevention.

SWE is considered to be less operator dependent and to have higher reproducibility than earlier ultrasound elastography techniques based on operator compression of the tissue to induce transient stress and assess tissue deformation (stiffness). A previous study showed that the reproducibility of YM measurements quantified by the interframe coefficient of variation was $22 \%$ within the vessel wall and $19 \%$ within the carotid plaque (23). Our study presented a preferable agreement (ICC $=0.911,95 \%$ CI, 0.848-0.948) for the assessment of PS using SWE between 2 independent sonographers.

SWE provides additional information related to plaque tissue stiffness, such as the presence of IPH and a large lipid core, which may indicate plaque vulnerability. Dahl et al. (27) reported that the stiffness of collagenous fiber was higher than that of lipids, and calcification had the highest stiffness among the plaque components. The mean YM values of representative plaques in the asymptomatic group were significantly higher than those in the symptomatic group $(78.5 \pm 25.4$ vs. $51.5 \pm 18.3 \mathrm{kPa})$. Ramnarine et al. (23) reported that the mean $\mathrm{YM}$ values of asymptomatic plaques and symptomatic plaques were 88 and $62 \mathrm{kPa}$, respectively, and these values were comparable to the findings obtained in our study. The regions of interest (ROIs) they selected were more than two circles each with 2-mm radii to sample the mean YM of the plaques randomly. Although the method can avoid the effects of the presence of different components on the mean YM, it also is associated with the problem of selection bias. In our study, we used the trace tool to evaluate the whole-plaque mean YM; thus, it might be able to avoid the issue of selection bias. Moreover, a significant relationship was evident between the YM of plaques and the degree of lumen narrowing. However, this relationship may be the result of a confounding factor as the severity of stenosis of symptomatic plaques was usually higher than that of asymptomatic plaques, and the mean YM of asymptomatic plaques was significantly higher than that of symptomatic plaques.

The assessment of the clinical potential of the advanced ultrasound techniques used in this study suggested that PS and IPN level were crucial markers for discriminating symptomatic plaques. SWE estimates of YM were a better marker than the IPN level, and combining the YM with IPN grades yielded improved discrimination performance (AUC $=0.89$ ). Therefore, compared with the degree of stenosis, the additional information provided by SWE and
SMI imaging in atherosclerotic vascular diseases may help to improve individual risk stratification.

Further studies are needed to validate our primary findings, to extend the study to quantitative analysis of IPN and to assess YM heterogeneity within plaques, which may further help to precisely identify vulnerable carotid plaques. Advances in the SMI technique should be developed to overcome the limitations of conventional Doppler ultrasound, which enables the visualization of IPN without the use of intravenous contrast. However, SMI in contrast to CEUS only can be assessed with a specific ultrasound system of Canon Aplio at present. The development of SWE technology, such as improving the frame rate, should improve the reliability and enable evaluation of temporal variation in tissue stiffness throughout the cardiac cycle. Our study design used clinical ischemic symptoms as a surrogate measure of vulnerable plaques. Large-scale multicenter studies with long-term follow-up are needed to assess the predictive performance and prognostic ability of our findings in identifying vulnerable plaques and the risk of stroke. These findings would provide the evidence required for implementation of SMI and SWE imaging techniques as routine vascular ultrasound examinations.

\section{Conclusions}

Advanced ultrasound techniques, including SWE and SMI, are able to identify features of vulnerable carotid plaques with preferable reproducibility and high specificity and sensitivity. Advanced ultrasound characteristics of vulnerable plaques are well correlated with patient ischemic symptoms. Prospective randomized trials are needed to validate that criteria observed with advanced ultrasound techniques are predictive of ischemic events and that advanced ultrasound techniques allow better patient selection for revascularization therapies.

\section{Acknowledgments}

We acknowledged all co-authors for their hard work of this study. We would like to thank American Journal Experts (AJE) for the help in polishing our paper.

Funding: This research was supported by the National Natural Science Foundation of China (ID 8173000716).

\section{Footnote}

Reporting Checklist: The authors have completed the STARD 
reporting checklist. Available at http://dx.doi.org/10.21037/ cdt-20-876

Data Sharing Statement: Available at http://dx.doi. org/10.21037/cdt-20-876

Peer Review File: Available at http://dx.doi.org/10.21037/ cdt-20-876

Conflicts of Interest: All authors have completed the ICMJE uniform disclosure form (available at http://dx.doi. org/10.21037/cdt-20-876). The authors have no conflicts of interest to declare.

Ethical Statement: The authors are accountable for all aspects of the work in ensuring that questions related to the accuracy or integrity of any part of the work are appropriately investigated and resolved. The study was conducted in accordance with the Declaration of Helsinki (as revised in 2013). The study was approved by the Institutional Review Board of the Beijing Tiantan Hospital (IRB No. KY2019-113-01). All participants gave written informed consent before ultrasound examinations.

Open Access Statement: This is an Open Access article distributed in accordance with the Creative Commons Attribution-NonCommercial-NoDerivs 4.0 International License (CC BY-NC-ND 4.0), which permits the noncommercial replication and distribution of the article with the strict proviso that no changes or edits are made and the original work is properly cited (including links to both the formal publication through the relevant DOI and the license). See: https://creativecommons.org/licenses/by-nc-nd/4.0/.

\section{References}

1. GBD 2016 Lifetime Risk of Stroke Collaborators, Feigin VL, Nguyen G, et al. Global, Regional, and CountrySpecific Lifetime Risks of Stroke, 1990 and 2016. N Engl J Med 2018;379:2429-437.

2. Kernan WN, Ovbiagele B, Black HR, et al. Guidelines for the prevention of stroke in patients with stroke and transient ischemic attack: a guideline for healthcare professionals from the American Heart Association/ American Stroke Association. Stroke 2014;45:2160-236.

3. Naylor AR, Ricco JB, de Borst GJ, et al. Management of Atherosclerotic Carotid and Vertebral Artery Disease: 2017 Clinical Practice Guidelines of the European Society for Vascular Surgery (ESVS). Eur J Vasc Endovasc Surg 2018;55:3-81.

4. Noguchi T, Yamada N, Higashi M, et al. High-intensity signals in carotid plaques on T1-weighted magnetic resonance imaging predict coronary events in patients with coronary artery disease. J Am Coll Cardiol 2011;58:416-22.

5. Demeure F, Bouzin C, Roelants V, et al. Head-to-Head Comparison of Inflammation and Neovascularization in Human Carotid Plaques: Implications for the Imaging of Vulnerable Plaques. Circ Cardiovasc Imaging 2017;10:e005846.

6. Naghavi M, Libby P, Falk E, et al. From vulnerable plaque to vulnerable patient: a call for new definitions and risk assessment strategies: Part I. Circulation 2003;108:1664-72.

7. Saba L, Saam T, Jäger HR, et al. Imaging biomarkers of vulnerable carotid plaques for stroke risk prediction and their potential clinical implications. Lancet Neurol 2019;18:559-72.

8. Villemain O, Correia M, Mousseaux E, et al. Myocardial Stiffness Evaluation Using Noninvasive Shear Wave Imaging in Healthy and Hypertrophic Cardiomyopathic Adults. JACC Cardiovasc Imaging 2019;12:1135-45.

9. Cosgrove D, Piscaglia F, Bamber J, et al. EFSUMB guidelines and recommendations on the clinical use of ultrasound elastography. Part 2: Clinical applications. Ultraschall Med 2013;34:238-53.

10. Naim C, Cloutier G, Mercure E, et al. Characterisation of carotid plaques with ultrasound elastography: feasibility and correlation with high-resolution magnetic resonance imaging. Eur Radiol 2013;23:2030-41.

11. Garrard JW, Ummur P, Nduwayo S, et al. Shear Wave Elastography May Be Superior to Greyscale Median for the Identification of Carotid Plaque Vulnerability: A Comparison with Histology. Ultraschall Med 2015;36:386-90.

12. Coli S, Magnoni M, Sangiorgi G, et al. Contrast-enhanced ultrasound imaging of intraplaque neovascularization in carotid arteries: correlation with histology and plaque echogenicity. J Am Coll Cardiol 2008;52:223-30.

13. Oura K, Kato T, Ohba H, et al. Evaluation of Intraplaque Neovascularization Using Superb Microvascular Imaging and Contrast-Enhanced Ultrasonography. J Stroke Cerebrovasc Dis 2018;27:2348-53.

14. Grant EG, Benson CB, Moneta GL, et al. Carotid artery stenosis: grayscale and Doppler ultrasound diagnosis-Society of Radiologists in Ultrasound consensus conference. Ultrasound Q 2003;19:190-8.

15. Touboul PJ, Hennerici MG, Meairs S, et al. Mannheim 
carotid intima-media thickness and plaque consensus (20042006-2011). An update on behalf of the advisory board of the $3 \mathrm{rd}$, 4th and 5 th watching the risk symposia, at the 13 th, 15th and 20th European Stroke Conferences, Mannheim, Germany, 2004, Brussels, Belgium, 2006, and Hamburg, Germany, 2011. Cerebrovasc Dis 2012;34:290-6.

16. Gray-Weale AC, Graham JC, Burnett JR, et al. Carotid artery atheroma: comparison of preoperative B-mode ultrasound appearance with carotid endarterectomy specimen pathology. J Cardiovasc Surg (Torino) 1988;29:676-81.

17. Zamani M, Skagen K, Scott H, et al. Carotid Plaque Neovascularization Detected With Superb Microvascular Imaging Ultrasound Without Using Contrast Media. Stroke 2019;50:3121-7.

18. Ramnarine KV, Garrard JW, Dexter K, et al. Shear wave elastography assessment of carotid plaque stiffness: in vitro reproducibility study. Ultrasound Med Biol 2014;40:200-9.

19. Gillard JH. Imaging of carotid artery disease: from luminology to function? Neuroradiology 2003;45:671-80.

20. Chen X, Wang H, Jiang Y, et al. Neovascularization in carotid atherosclerotic plaques can be effectively evaluated by superb microvascular imaging (SMI): Initial experience. Vasc Med 2020;25:328-33.

21. Zhu YC, Jiang XZ, Bai QK, et al. Evaluating the Efficacy

Cite this article as: Li Y, Zheng S, Zhang J, Wang F, Liu X, $\mathrm{He} W$. Advance ultrasound techniques for the assessment of plaque vulnerability in symptomatic and asymptomatic carotid stenosis: a multimodal ultrasound study. Cardiovasc Diagn Ther 2021;11(1):28-38. doi: 10.21037/cdt-20-876 of Atorvastatin on Patients with Carotid Plaque by an Innovative Ultrasonography. J Stroke Cerebrovasc Dis 2019;28:830-7.

22. Yang DB, Zhou J, Feng L, et al. Value of superb microvascular imaging in predicting ischemic stroke in patients with carotid atherosclerotic plaques. World J Clin Cases 2019;7:839-48.

23. Ramnarine KV, Garrard JW, Kanber B, et al. Shear wave elastography imaging of carotid plaques: feasible, reproducible and of clinical potential. Cardiovasc Ultrasound 2014;12:49.

24. Xu J, Lu X, Shi GP. Vasa vasorum in atherosclerosis and clinical significance. Int J Mol Sci 2015;16:11574-1608.

25. Fleiner M, Kummer M, Mirlacher M, et al. Arterial neovascularization and inflammation in vulnerable patients: early and late signs of symptomatic atherosclerosis. Circulation 2004;110:2843-50.

26. van den Oord SC, Akkus Z, Bosch JG, et al. Quantitative contrast-enhanced ultrasound of intraplaque neovascularization in patients with carotid atherosclerosis. Ultraschall Med 2015;36:154-61.

27. Dahl JJ, Dumont DM, Allen JD, et al. Acoustic radiation force impulse imaging for noninvasive characterization of carotid artery atherosclerotic plaques: a feasibility study. Ultrasound Med Biol 2009;35:707-16. 